\title{
Extending the Construct Network of Trait Disinhibition to the Neuroimaging Domain: Validation of a Bridging Scale for Use in the European IMAGEN Project
}

\author{
Sarah J. Brislin', Christopher J. Patrick', Herta Flor ${ }^{2,3}$, Frauke Nees ${ }^{2}$, \\ Angela Heinrich ${ }^{2}$, Laura E. Drislane ${ }^{4}$, James R. Yancey', Tobias Banaschewski ${ }^{2}$, \\ Arun L. W. Bokde ${ }^{5}$, Uli Bromberg ${ }^{6}$, Christian Büchel ${ }^{6}$, Erin Burke Quinlan 7 , \\ Sylvane Desrivières ${ }^{7}$, Vincent Frouin ${ }^{8}$, Hugh Garavan ${ }^{9}$, Penny Gowland ${ }^{10}$, \\ Andreas Heinz " , Bernd Ittermann '2, Jean-Luc Martinot 13,14,15,16, \\ Marie-Laure Paillère Martinot ${ }^{16}$, Dimitri Papadopoulos Orfanos ${ }^{8}$ iD, \\ Luise Poustka $^{2,17}$, Juliane H. Fröhner ${ }^{18}$ (D), Michael N. Smolka ${ }^{18}$, Henrik Walter ${ }^{11}$, \\ Robert Whelan ${ }^{19}$, Patricia Conrod ${ }^{20}$, Argyris Stringaris ${ }^{21}$, Maren Struve ${ }^{2}$, \\ Betteke van Noort "', Yvonne Grimmer ${ }^{2}$, Tahmine Fadai ${ }^{6}$, Gunter Schumann, \\ and Jens Foell' (iD
}

\begin{abstract}
Trait disinhibition, a clinical-liability construct, has well-established correlates in the diagnostic, self-rating, task-behavioral, and brain potential response domains. Recently, studies have begun to test for neuroimaging correlates of this liability factor, but more work of this type using larger data sets is needed to clarify its brain bases. The current study details the development and validation of a scale measure of trait disinhibition composed of questionnaire items available in the IMAGEN project, a large-scale longitudinal study of factors contributing to substance abuse that includes clinical interview, self-report personality, task-behavioral, neuroimaging, and genomic measures. Using a construct-rating and psychometric refinement approach, a scale was developed that evidenced: (a) positive relations with interview-assessed psychopathology in the IMAGEN sample, both concurrently and prospectively and (b) positive associations with scale measures of disinhibition and reported psychopathology, and a robust negative correlation with P3 brain response, in a separate adult sample $\left(M_{\text {age }}=19.5\right)$. These findings demonstrate that a common scale measure can index this construct from adolescence through to early adulthood, and set the stage for systematic work directed at identifying neural and genetic biomarkers of this key liability construct using existing and future data from the IMAGEN project.
\end{abstract}

\section{Keywords}

externalizing psychopathology, trait disinhibition, liability, neuroimaging, IMAGEN

Research initiatives directed at clarifying the neural mechanisms that give rise to clinically significant dysfunction have led to an increased need for innovative methods for indexing psychological traits. The National Institute of Mental Health's Research Domain Criteria initiative (Kozak \& Cuthbert, 2016) calls for integrating variables from the neurophysiological domain with more standard assessment measures to move the field toward a neurobiologically oriented science of psychopathology. Similar calls have been made by the National Institute on Alcohol Abuse and Alcoholism (Kwako, Momenan, Litten, Koob, \& Goldman, 2016) and the National Research Council (2015). However, there are significant challenges that must be addressed in attempting to assess psychopathology-relevant constructs using neurophysiological measures (Patrick \& Hajcak, 2016; Patrick, Venables, et al., 2013).

In this article, we report results from new work we have undertaken to advance efforts in this direction. We first describe the development and initial validation of a scale measure of the neurobehavioral trait construct of inhibitory control (inhibition-disinhibition) using self-report data from the European IMAGEN project (Schumann et al., 2010), a large-scale longitudinal risk study that includes measures from multiple domains including neuroimaging. 
Using data for a separate participant sample, we then show that scores on this IMAGEN-Disinhibition (IMAGENDIS) scale covary significantly with an established brain indicator of disinhibitory tendencies, the oddball-P3 response. This work demonstrates that a self-report scale linked to an emerging cross-domain measurement model of inhibition-disinhibition (Patrick, Venables, et al., 2013; Venables et al., in press) shows expected relations with brain reactivity. More broadly, the current work creates a foundation for further research using IMAGEN study data to identify robust neuroimaging correlates of trait disinhibition that relate in turn to task-behavioral and clinical-diagnostic variables, as a step toward a cross-domain, "neuroclinical" assessment protocol (Kwako et al., 2016) for disinhibitory liability.

\section{Cross-Domain Conceptualization of Trait Disinhibition}

Inhibitory control, the focus of the current work, has been conceptualized in both behavioral-process terms (e.g., as "response inhibition" Kozak \& Cuthbert, 2016) and in individual-difference terms (e.g., as "externalizing proneness"; Krueger, Markon, Patrick, Benning, \& Kramer, 2007 or "trait disinhibition"; Yancey, Venables, Hicks, \& Patrick, 2013; Young et al., 2009). Our emphasis here is on the latter conceptualization - in particular, on a dispositional construct of inhibition-disinhibition that has been viewed as a liability factor for substance problems (Pihl, Peterson, \& Finn, 1990), child disruptive disorders (Barkley, 1997), adult antisocial behavior (Morgan \& Lilienfeld, 2000), and problems of these types in general (Krueger et al., 2002;
Young et al., 2000). Historically, traits have been viewed as general biobehavioral propensities that influence specific responses across different situations (e.g., Allport, 1937; Eysenck, 1960; Tellegen, 1985). Consistent with this, we conceive of trait disinhibition as a latent attribute that influences self-reported tendencies, affects neural responses during cognitive processing, and increases risk for impulsecontrol (externalizing) problems. This concept of trait disinhibition forms the basis for our hypothesis that this attribute can be assessed through self-report in a way that correlates with brain reactivity.

Recent efforts to operationalize trait disinhibition have provided evidence that this dispositional construct can provide an effective bridge for interfacing neural response variables with clinical diagnoses. For example, reliable associations with both impulse-disorder diagnoses and neural reactivity measures (Patrick, Venables, et al., 2013) including the best-established brain response indicator of externalizing problems, the oddball-P3 response-were found for a disinhibition scale composed of items from the Externalizing Spectrum Inventory (ESI-Disinhibition; Patrick, Kramer, Krueger, \& Markon, 2013). The P3 is a positive polarity event-related potential (ERP), maximal over parietal scalp sites, that peaks between 300 and $600 \mathrm{~ms}$ following the onset of salient stimuli within a task. Reduced amplitude of P3 response to infrequent ('oddball') target stimuli within a target/nontarget task series has consistently been shown to be associated with externalizing psychopathology (Begleiter, Porjesz, Bihari, \& Kissin, 1984; Iacono et al., 2002; Nelson, Patrick, \& Bernat, 2011; Patrick et al., 2006). Because of this, P3 has been championed as a neurophysiological marker of disinhibitory liability. ${ }^{1}$ Importantly,

\footnotetext{
'Florida State University, Tallahassee, FL, USA

${ }^{2}$ Heidelberg University, Mannheim, Germany

${ }^{3}$ University of Mannheim, Mannheim, Germany

${ }^{4}$ University of Michigan, Ann Arbor, MI, USA

${ }^{5}$ The University of Dublin, Dublin, Ireland

${ }^{6}$ University Medical Centre Hamburg-Eppendorf, Hamburg, Germany

${ }^{7}$ King's College London, London, England, UK

${ }^{8}$ Université Paris-Saclay, Gif-sur-Yvette, France

'University of Vermont, Burlington, VT, USA

${ }^{10}$ University of Nottingham, University Park, Nottingham, UK

"Charité-Universitätsmedizin Berlin, Berlin, Germany

${ }^{12}$ Physikalisch-Technische Bundesanstalt, Braunschweig, Germany

${ }^{13}$ University Paris Sud, Orsay, France

${ }^{14}$ University Paris Descartes, Paris, France

${ }^{15}$ Université Paris-Sorbonne, Paris, France

${ }^{16}$ Maison de Solenn, Cochin Hospital, Paris, France

${ }^{17}$ Medical University of Vienna, Vienna, Austria

${ }^{18}$ Technische Universität Dresden, Dresden, Germany

${ }^{19}$ University College Dublin, Dublin, Ireland

${ }^{20}$ Université de Montréal, Quebec, Canada

${ }^{21}$ National Institute of Mental Health, Bethesda, MD, USA
}

Corresponding Author:

Sarah J. Brislin, Department of Psychology, Florida State University, I 107 West Call Street, Tallahassee, FL 32306-430 I, USA.

Email: brislin@psy.fsu.edu 
research has shown that the relationship between $\mathrm{P} 3$ response and externalizing psychopathology mainly reflects shared genetic influences (Hicks et al., 2007) and that the ESIDisinhibition scale indexes genetic variance in common between P3 amplitude (Yancey et al., 2013) and externalizing disorders.

Recent studies have also found that ESI-Disinhibition scores relate to brain reactivity as indexed by functional magnetic resonance imaging. Foell et al. (2016) reported that the ESI-Disinhibition scale predicted reactivity in subcortical brain structures when anticipating and viewing emotional images, and Abram et al. (2015) found that individuals high in trait disinhibition demonstrated deviant patterns of resting-state brain activity. Together with findings for oddball-P3 and other ERP variables (Hall et al., 2007; Nelson et al., 2011; Patrick, Venables, et al., 2013), these results provide empirical support for trait disinhibition as a construct that can bridge between psychopathology and neurophysiology. However, neuroimaging research with larger samples is needed to extend the construct network for trait disinhibition as it currently stands (Patrick, Venables, et al., 2013; see also Venables et al., in press) into the domain of brain circuits and processes.

\section{The IMAGEN Project}

As discussed in writings on the Research Domain Criteria research framework (e.g., Kozak \& Cuthbert, 2016), a detailed understanding of how neural processes relate to psychological phenomena and behavior requires multilevel data sets that (a) include self-report, behavioral, and physiological measures and (b) contain a large enough number of participants and measures to allow for well-powered multivariate analyses. One such study is the IMAGEN project (Schumann et al., 2010), a large-scale consortium study in which data are being collected for over 2,000 participants at 2 -year intervals across eight European test sites. Assessment measures include clinical interviewing, behavioral and neuropsychological testing, functional and structural neuroimaging, and genotyping, along with administration of self-report questionnaires to index traits and characteristics hypothesized to relate to externalizing behavior and substance use. Data from the first two waves of the IMAGEN project have already served as the basis for numerous published reports focusing on genetic, physiological, and behavioral aspects of externalizing psychopathology, with a third wave of data collection to occur during 2017.

Besides providing access to neuroimaging data along with other measures for a uniquely large sample, the IMAGEN project also presents opportunities to explore and clarify the progression of trait disinhibition across time, from premorbid liability to active expression of psychopathology. Relevant to this, existing longitudinal studies have provided evidence that clinical problems in adulthood reflect extensions of dispositional liabilities evident at earlier ages (Kim-Cohen et al., 2003; Krueger, 1999; Young et al., 2009). With this in mind, the current study was also undertaken to evaluate whether trait disinhibition as it has been conceptualized and measured in adult samples shows validity within a younger aged adolescent sample, as a basis for further investigations of the developmental course and correlates of this liability factor.

While the IMAGEN project database includes selfreport measures of attributes related to impulse-control problems, the study does not include a specific scale for indexing trait disinhibition as represented in the ESI model. However, reliable and valid scales for assessing trait disinhibition conceptualized in this way can be constructed from items of already-existing questionnaire measures. For example, Brislin, Drislane, Smith, Edens, and Patrick (2015) developed a scale for indexing trait disinhibition using items from the Multidimensional Personality Questionnaire (MPQ; Tellegen, 1982), and validated this scale using data from healthy community participants and incarcerated criminal offenders. This scale development method has also been used to create effective scale measures of disinhibition from items of the Minnesota Multiphasic Personality Inventory-2 (Sellbom et al., 2016) and the Psychopathic Personality Inventory (Hall et al., 2014). The current study presents results from a new application of this scale-development method, as this approach has not previously been used to create a measure of disinhibition using item data from adolescents.

\section{Current Aims and Hypotheses}

The primary aims of the current study were to: (a) evaluate the effectiveness of items administered as part of the IMAGEN study protocol for indexing trait disinhibition, by developing a proxy scale measure of this liability construct; (b) assess, within the IMAGEN sample, the scale's internal reliability and external validity in terms of relations with clinical criterion measures, both concurrently (at ages 14 and 16 years) and prospectively (from age 14 to age 16 years); and (c) further examine, within an independent sample of adult participants, the scale's reliability and validity in relation to neurophysiological (P3 brain response) as well as clinical (reported problems) criterion measures.

Following procedures used to develop trait disinhibition scales from other item sets (e.g., Brislin et al., 2015; Hall et al., 2014), candidate items were first evaluated for construct relevance using a consensus-rating approach, and then refined through internal psychometric analyses. Based on known psychological correlates of externalizing proneness and trait disinhibition (Patrick \& Drislane, 2015; Patrick, Fowles, \& Krueger, 2009), we hypothesized that items represented in the following set of IMAGEN study scales would provide good coverage of relevant psychological content 
and thus serve as an effective item pool for constructing a valid scale measure of trait disinhibition: Neuroticism Extraversion Openness-Five Factor Inventory (NEOFFI)-Conscientiousness and Agreeableness subscales; Temperament and Character Inventory-Revised (TCI-R) Novelty-Seeking Scale-Impulsiveness, Exploratory Excitability, Disorderliness, and Extravagance subscales; Substance Use Risk Profile Scale (SURPS)-Impulsivity and Sensation Seeking subscales; Strengths and Difficulties Questionnaire (SDQ) - Behavior Problems, Peer Problems, and Emotional Dysregulation subscales; and Life Events Questionnaire (LEQ) - items pertaining to past instances of rule-breaking behavior.

Within the IMAGEN study sample, we hypothesized that scores on the IMAGEN-DIS proxy scale would be stable over time and would demonstrate robust convergence with clinical ratings of antisocial and impulsive behavior, including conduct disorder (CD), oppositional defiant disorder (ODD), and attention deficit hyperactivity disorder (ADHD). In addition, we hypothesized that scores on the IMAGENDIS scale would be positively associated with self-reported alcohol and drug use/abuse. Last, based on the concept of trait disinhibition as an underlying liability factor for impulse-control problems, we hypothesized that IMAGENDIS scores from the initial project assessment (age 14 years) would prospectively predict to later (age 16 years) clinical ratings of antisocial and impulsive behaviors.

To further examine the reliability, validity, and developmental generalizability of the IMAGEN-DIS scale as a proxy for existing self-report measures of trait disinhibition, the current study also examined the psychometric properties and convergent and discriminant validity of the IMAGEN-DIS scale in an adult undergraduate sample. We hypothesized that scores on the IMAGEN-DIS scale would show good psychometric properties and strong convergence with the General ESI-Disinhibition scale, as well as associations with selfreport measures of substance abuse, antisocial behavior, and externalizing-related personality traits as indexed by the MPQ (i.e., traits from domains of Negative Emotionality and [low] Constraint). With the aim of linking the new scale measure to established neurophysiological findings for trait disinhibition, we hypothesized that scores on the IMAGEN-DIS scale would be associated with reduced amplitude of P3 response to target stimuli in a visual oddball task.

\section{Study I: Scale Development and Validation Within the IMAGEN Sample}

\section{Method}

Participants. Scale development and initial validation were undertaken with data for a sample of adolescents, recruited and tested through the IMAGEN consortium project (see
Schumann et al., 2010, for a detailed description of the project sample and recruitment strategy). The current study utilized all participants with complete self-report, diagnostic interview, and parent report data. This resulted in an analysis sample of 2,186 adolescents ( $M_{\text {age }}=14.4 ; 50.6 \%$ female). In the context of the longitudinal design of the IMAGEN project, data were collected from this sample of participants 2 years later. Due to participant attrition $(\sim 20 \%)$, data for the same self-report, interview, and parent report measures were collected for a subset of the original sample at Time 2 $\left(n=1,709 ; M_{\text {age }}=16.5\right)$.

Procedure. In the Time 1 IMAGEN assessment that was used for scale development and initial validation, questionnaire and interview measures were administered in person as part of a larger study protocol that included semistructured interviews and functional magnetic resonance imaging test sessions with the adolescent participant, and interviews with a parent. For the Time 2 IMAGEN assessment, a reduced remote protocol was used in which participants were interviewed over the phone and filled out questionnaires online. At each assessment time, participants were provided with information about the study, and parents and adolescents provided written consent and assent, respectively.

Measures Used as Sources of Candidate Items. The approach to scale development using IMAGEN study data followed procedures that have been used previously to construct scale measures of trait disinhibition from items of existing inventories (e.g., Brislin et al., 2015). Selected items from self-report inventories containing disinhibition-relevant content were used as the pool of candidate items, from which the resultant IMAGEN-DIS scale was derived. All measures used as sources of candidate items were administered on a computer, either in the lab at Time 1 or online at Time 2.

Neuroticism Extraversion Openness-Five Factor Inventory (Costa \& McCrae, 1992). The NEO-FFI is a 60-item selfreport questionnaire developed to assess the personality dimensions of the five-factor model of personality. This instrument is an abbreviated version of the 240-item revised NEO Personality Inventory (Costa \& McCrae, 1992), consisting of items that index the broad trait domains of the five-factor model. Participants responded to items on a 5-point Likert-type scale ranging from strongly disagree to strongly agree.

Temperament and Character Inventory-Revised (Cloninger, 1999) Novelty-Seeking Scale. The Novelty-Seeking scale of the TCI-R includes four subscales: Exploratory Impulsiveness ( 9 items), Disorderliness ( 7 items), Excitability (10 items), and Extravagance (9 items). Participants responded to items 
of this measure on a 5-point Likert-type scale ranging from definitely false to definitely true.

Substance Use Risk Profile Scale (Conrod \& Woicik, 2002; Woicik, Stewart, Pihl, \& Conrod, 2009). The SURPS is a 23-item self-report questionnaire developed to assess personality traits that have been found to confer risk for substance use and misuse. This measure assesses four distinct and largely independent personality dimensions: Anxiety Sensitivity (five items), Hopelessness (seven items), Sensation Seeking (six items), and Impulsivity (five items). Participants responded to items on a 4-point Likert-type scale ranging from strongly disagree to strongly agree.

Strengths and Difficulties Questionnaire (Goodman, 200I). The SDQ is a 25-item questionnaire developed to assess psychopathology in adolescents. Participants rated items pertaining to their expression of Hyperactivity/Inattention, Behavior Problems, Peer Problems, Emotional Dysregulation, and their General Psychosocial Well-Being on a 3-point Likert-type scale ranging from not true to certainly true.

Life Events Questionnaire (adapted from Newcomb, Huba, \& Bentler, 198I). The LEQ is a 39-item self-report measure that asks respondents about the occurrence of positive (e.g., Met a teacher I liked a lot) and negative (e.g., Death in the family) life events over the entire life span and over the past year.

\section{Criterion Measures}

Clinician Rating. The interview-based Development and Well-Being Assessment (DAWBA; Goodman, Ford, Richards, Gatward, \& Meltzer, 2000) was administered to adolescents to assess for the presence of internalizing and externalizing psychopathology according to criteria specified in the fourth edition of the Diagnostic and Statistical Manual of Mental Disorders (DSM-IV; American Psychiatric Association, 2000). Participants responded to both open and closed questions, and diagnostic ratings were subsequently made through clinical consensus. Participants were designated as either meeting criteria for the disorder, meeting some but not all criteria, or not meeting criteria for the disorder.

\section{Self-Report}

Alcohol Use Disorders Identification Test (AUDIT; Allen, Litten, Fertig, \& Babor, 1997). The AUDIT was administered to adolescent participants as part of an online questionnaire battery. Participants responded to questions about the frequency and severity of their alcohol use. This measure yields scores on three subscales: Frequency (three items; Time $1 \alpha=.69$; Time $2 \alpha=.75$ ), Alcohol Dependence
Symptoms (three items; Time $1 \alpha=.56$; Time $2 \alpha=.40$ ), and Harmful Alcohol Use (four items; Time $1 \alpha=.49$; Time $2 \alpha=.52$ ). Scores on these three subscales are computed as sums of responses to individual items within each. A total score was also calculated, reflecting the sum across the three subscales (Time $1 \alpha=.72$; Time $2 \alpha=.75$ ).

European School Survey Project on Alcohol and Drugs (ESPAD). The ESPAD is a self-report measure consisting of questions regarding use of illicit substances that was administered to participants as part of an online questionnaire battery. For purposes of the current study, variables reflecting lifetime use of marijuana and lifetime use of all other drugs (each coded yes/no) were examined as criterion variables.

Externalizing Composite (EXT). Based on comorbidity research demonstrating that $\mathrm{CD}$, alcohol dependence, and drug dependence cohere together into a common externalizing spectrum (Krueger et al., 2002) or class (Vaidyanathan, Patrick, \& Iacono, 2011), a composite score reflecting overall externalizing psychopathology was computed as the mean of standardized scores for the DAWBA CD rating, AUDIT Total Alcohol Use score, and combined ESPAD Marijuana and Other Substance variables. Scores for this composite were computed at Time 1 and Time 2.

Scale Construction and Validation. Construction of the IMAGEN-DIS scale occurred in three phases, described in more detail below. In the development phase, candidate items from the NEO-FFI, TCI, LEQ, SURPS, and SDQ were selected based on ratings of construct relevance. Next, the items selected for potential inclusion underwent refinement based on internal psychometric analyses. Then, classical test theory statistics were used to evaluate the psychometric properties of the final item set for the scale. All analyses performed in the refinement phase utilized the data only from the Time 1 IMAGEN assessment, when participants were approximately 14.4 years old.

Development phase and Candidate Item Scale construction. Two clinical psychology graduate students and two $\mathrm{PhD}$ psychologists provided ratings of construct relevancy for the initial pool of 187 items. Raters were provided with a written definition of the construct of disinhibition (for a copy, see online Supplement) used in prior studies (e.g., Brislin et al., 2015) and judged the degree to which each item related to disinhibition, answering the question "To what extent does this item represent disinhibition?" The raters selected one of five choices for each item: unrelated to disinhibition, strongly represents HIGH disinhibition, somewhat represents HIGH disinhibition, somewhat represents LOW disinhibition, and strongly represents LOW disinhibition. Candidate items for the Disinhibition scale were then identified based on the level of agreement for particu- 
Table I. List of Items and Psychometric Properties for the Items Comprising the Final 22-Item IMAGEN Disinhibition (IMAGEN-DIS) Scale.

\begin{tabular}{|c|c|c|}
\hline $\begin{array}{l}\text { Source (item } \\
\text { number) }\end{array}$ & $\begin{array}{l}\text { Adjusted item } \\
\text { total correlation }\end{array}$ & Item category \\
\hline \multicolumn{3}{|l|}{ NEO-FFI } \\
\hline 9 & .35 & Agreeableness \\
\hline $20^{\mathrm{a}}$ & .32 & Conscientiousness \\
\hline $40^{\mathrm{a}}$ & .31 & Conscientiousness \\
\hline 45 & .40 & Conscientiousness \\
\hline 55 & .42 & Conscientiousness \\
\hline \multicolumn{3}{|l|}{ TCl-R } \\
\hline I & .25 & Exploratory Excitability \\
\hline $14^{\mathrm{a}}$ & .20 & Extravagance \\
\hline 24 & .37 & Extravagance \\
\hline 44 & .33 & Disorderliness \\
\hline $47^{\mathrm{a}}$ & .34 & Sensation Seeking \\
\hline 109 & .56 & Disorderliness \\
\hline 122 & .28 & Exploratory Excitability \\
\hline \multicolumn{3}{|l|}{ SURPS } \\
\hline 2 & .36 & Impulsivity \\
\hline II & .51 & Impulsivity \\
\hline 15 & .26 & Impulsivity \\
\hline 16 & .41 & Sensation Seeking \\
\hline \multicolumn{3}{|l|}{ SDQ } \\
\hline Reflects $^{\mathrm{a}}$ & .43 & Problematic Impulsivity \\
\hline Steals & .25 & Theft \\
\hline Tantrum & .26 & Alienation \\
\hline \multicolumn{3}{|l|}{ LEQ } \\
\hline 4 & .19 & Rebelliousness \\
\hline 5 & .16 & Theft \\
\hline 19 & .28 & Rebelliousness \\
\hline
\end{tabular}

Note. $N=2,186$. NEO-FFI = Neuroticism Extraversion Openness-Five Factor Inventory; $\mathrm{TCl}-\mathrm{R}=$ Temperament and Character InventoryRevised; SURPS = Substance Use Risk Profile Scale; SDQ = Strengths and Difficulties Questionnaire; LEQ = Life Events Questionnaire. ${ }^{a}$ Items are reversed.

lar items across raters. Items that had been rated as strongly representing high or low disinhibition by at least three of the four raters were selected as initial scale indicators. There were 14 items that met this initial criterion. These items had different response formats; therefore, scores for these candidate items were scaled to a common 5-point metric (0-4) to allow for aggregation into a total score.

Refinement phase. Following the identification of highly relevant items, the provisional 14-item Disinhibition scale was refined by examining adjusted item-total $r$ s for candidate items. One candidate item was removed based on this criterion, which improved scale homogeneity (Cronbach's alpha). Next, additional items that had been rated as strong indicators of high or low disinhibition by at least two of the four raters, and somewhat indicative of the construct by the remaining two raters, were evaluated for possible inclusion. Items meeting this criterion that showed strong item-total correlations with the revised (13-item) provisional scale, and that added to the internal consistency of the scale, were retained. Nine additional items were included in the scale. Thus, the refinement phase resulted in a 22-item Disinhibition scale, which included 17 items worded in the direction of high disinhibition and 5 items worded in the direction of low disinhibition.

External validation. Following the refinement phase, the construct validity of the final IMAGEN-DIS scale was evaluated by computing Pearson correlation coefficients for the IMAGEN-DIS scale at Time 1 and Time 2, with itself and with questionnaire- and interview-based criterion variables as described above. In the undergraduate sample, the convergent and discriminant validity of the IMAGEN-DIS scale was evaluated using correlational analyses to examine associations between the scale and self-report-based criterion measures. In addition, the validity of the scale was evaluated in this sample by examining its association with target $\mathrm{P} 3$ response from the visual oddball task.

\section{Results}

Scale Content and Properties. The final 22-item IMAGEN-DIS scale (see Table 1) consists of items from the five inventories described above, and includes five reverse-keyed items. Though modest in length and diverse in terms of item content, the final IMAGEN-DIS scale demonstrated acceptable to good internal consistency (Time $1 \alpha=.78$; Time $2 \alpha=.81$ ). In the subsample of participants for whom data were available for both Time 1 and Time $2(n=1,585)$, the IMAGEN-DIS scale also evidenced good temporal stability, with scale scores at Time 1 showing a strong, significant association with scores at Time $2(r=.61, p<.001)$.

External Validation. Results for analyses examining associations between IMAGEN-DIS scale scores at assessment Times 1 and 2 and clinical ratings based on participant responses to questions on the DAWBA at each time point are presented in Table 2. Consistent with hypotheses, IMAGEN-DIS scores demonstrated robust associations with clinical ratings performed at the same time: Time 1 IMAGEN-DIS scores showed modest associations with Time 1 clinician ratings of $\mathrm{CD}$, ODD, $\mathrm{ADHD}$, but, consistent with hypotheses, were unrelated to ratings of major depression (MDD) and generalized anxiety disorder. Similarly, at Time 2 , clinician ratings of $\mathrm{CD}, \mathrm{ODD}, \mathrm{ADHD}$, and MDD were significantly associated with IMAGEN-DIS scores collected at the same time point, while generalized anxiety disorder ratings were unrelated. 
Table 2. Correlations for IMAGEN-DIS Scores with DAWBA Diagnostic Ratings Within and Across Assessment Times I ( $M_{\text {age }}$ $=14.4)$ and $2\left(M_{\text {age }}=16.5\right)$.

\begin{tabular}{|c|c|c|}
\hline Criterion measure & $\begin{array}{l}\text { Disinhibition } \\
\text { (Time I) }\end{array}$ & $\begin{array}{l}\text { Disinhibition } \\
\text { (Time 2) }\end{array}$ \\
\hline \multicolumn{3}{|l|}{ DAWBA (Time I; $n=1,258$ ) } \\
\hline Conduct disorder & $.16 * *$ & $.10 * *$ \\
\hline Oppositional defiant disorder & $.13^{* *}$ & $.11 * *$ \\
\hline ADHD & $.12^{* *}$ & $.08^{*}$ \\
\hline Major depression & .06 & .04 \\
\hline Generalized anxiety disorder & .03 & .02 \\
\hline \multicolumn{3}{|l|}{ DAWBA (Time 2; $n=1,452$ ) } \\
\hline Conduct disorder & $.14 * *$ & $.18^{* *}$ \\
\hline Oppositional defiant disorder & $.09 * *$ & $.12^{* *}$ \\
\hline ADHD & $.17 * *$ & $.20 * *$ \\
\hline Major depression & $.08 *$ & $.10 * *$ \\
\hline Generalized anxiety disorder & .02 & .01 \\
\hline
\end{tabular}

Note. IMAGEN-DIS = IMAGEN-Disinhibition; DAWBA = Development and Well-Being Assessment; ADHD = attention deficit hyperactivity disorder; Times I and 2 = first and second IMAGEN project assessments. Prospective validity coefficients (i.e., for prediction of Time 2 diagnostic ratings from Time I IMAGEN-DIS scale scores) are in bold.

$*_{p}<.0 \mathrm{I} . * * p<.00 \mathrm{I}$.

Table 3. Correlations for Self-Report Substance Use Variables with Scores on the IMAGEN-DIS Scale at Time I $\left(M_{\text {age }}=14.4\right)$ and Time $2\left(M_{\text {age }}=16.5\right)$.

\begin{tabular}{|c|c|c|}
\hline Criterion measure & $\begin{array}{c}\text { Disinhibition } \\
\text { (Time I) }\end{array}$ & $\begin{array}{c}\text { Disinhibition } \\
\text { (Time 2) }\end{array}$ \\
\hline \multicolumn{3}{|l|}{ AUDIT (Time I; $n=1,599$ ) } \\
\hline Total & $.37^{* *}$ & $.26 * *$ \\
\hline Frequent and hazardous use & $.36 * *$ & $.25^{* *}$ \\
\hline Alcohol dependence & $.25^{* *}$ & $.17^{* *}$ \\
\hline Harmful alcohol use & $.29 * *$ & $.19 * *$ \\
\hline \multicolumn{3}{|l|}{ ESPAD (Time I; $n=1,60 I$ ) } \\
\hline Lifetime marijuana & $.24 * *$ & $.15^{* *}$ \\
\hline Lifetime other drug & $.17^{* *}$ & $.14 * *$ \\
\hline \multicolumn{3}{|l|}{ AUDIT (Time 2; $n=1,549$ ) } \\
\hline Total & $.28 * *$ & $.42^{* *}$ \\
\hline Frequent and hazardous use & $.27 * *$ & $.40 * *$ \\
\hline Alcohol dependence & $.13 * *$ & $.23 * *$ \\
\hline Harmful alcohol use & $.17 * *$ & $.30 * *$ \\
\hline \multicolumn{3}{|l|}{ ESPAD (Time $2 ; n=1,550$ ) } \\
\hline Lifetime marijuana & $.24 * *$ & $.31 * *$ \\
\hline Lifetime other drug & $.12 * *$ & $.24 * *$ \\
\hline
\end{tabular}

Note. IMAGEN-DIS = IMAGEN-Disinhibition; AUDIT = Alcohol Use Disorders Identification Test; ESPAD = European School Survey Project on Alcohol and Drugs; Time I and Time 2 = first and second IMAGEN project assessments. Prospective validity coefficients (i.e., for prediction of Time 2 diagnostic ratings from Time I IMAGEN-DIS scale scores) are in bold.

$* p<.01 . * * p<.001$.
The IMAGEN-DIS scale also showed prospective validity in terms of associations for IMAGEN-DIS at Time 1 with clinical ratings at Time 2 (see Table 2). Specifically, IMAGEN-DIS at Time 1 was correlated significantly with clinician ratings of $\mathrm{CD}, \mathrm{ODD}, \mathrm{ADHD}$, and MDD at Time 2. Regression analyses were run to further examine prospective prediction. For ratings of CD at Time 2, IMAGEN-DIS at Time 1 was still a significant predictor $(\beta=.10, p<.01)$ even when controlling for $\mathrm{CD}$ ratings at Time 1. Similarly, IMAGEN-DIS significantly predicted $(\beta=.13, p<.001)$ ADHD ratings at Time 2 above and beyond ADHD ratings at Time 1 . The association between ODD ratings at Time 2 and IMAGEN-DIS at Time 1 decreased, but only slightly below significance, when controlling for ODD ratings at Time $1(\beta=.06, p<.05)$.

Results for analyses examining associations between IMAGEN-DIS and self-report measures of alcohol and substance use at Time 1 and Time 2 are presented in Table 3 . Consistent with hypotheses and the pattern of associations with clinical ratings, IMAGEN-DIS scores were most strongly associated with self-report measures collected at the same time. In addition, associations between IMAGENDIS and substance use assessed concurrently at Time 2 were stronger than those between IMAGEN-DIS and substance use assessed at Time 1. As hypothesized, across data collection (Time 1 and Time 2), IMAGEN-DIS scale scores were significantly associated with all self-report substance use variables, indicating good convergent validity for the scale. In addition, the IMAGEN-DIS scale evidenced prospective validity, demonstrating associations between IMAGEN-DIS at Time 1 and self-report of alcohol and substance use at Time 2 (Table 3).

The prospective validity of the IMAGEN-DIS scale in predicting alcohol and substance use was also examined using regression analyses. AUDIT Total score at Time 2 was significantly predicted by IMAGEN-DIS at Time 1 ( $\beta=.17, p<.001$ ), even when accounting for AUDIT Total score at Time 1. Similarly, IMAGEN-DIS at Time 1 significantly predicted lifetime marijuana use at Time $2(\beta=.17$, $p<.001)$ and lifetime other drug use at Time $2(\beta=.08$, $p<.01)$, when controlling for scores on these drug variables at Time 1.

Associations between IMAGEN-DIS and the EXT score were examined using bivariate correlations. IMAGEN-DIS at Time 1 was significantly correlated with EXT at Time 1 $(r=.37, p<.001)$ and EXT at Time $2(r=.29, p<.001)$. Similarly, at Time 2, IMAGEN-DIS was significantly associated with EXT at Time $2(r=.41, p<.001)$. With regard to prospective prediction, IMAGEN-DIS at Time 1 significantly predicted EXT at Time $2\left(\beta=.16, s r^{2}=.02, p<.001\right.$, $95 \%$ confidence interval $[.25, .47])$, over and above scores on EXT at Time 1. 


\section{Study 2: Replication and Extension in an Undergraduate Sample}

\section{Method}

Items comprising the IMAGEN-DIS scale were administered to a separate undergraduate sample $\left(n=66 ; M_{\text {age }}=\right.$ $19.5 ; 57.6 \%$ female) as part of a larger testing protocol that included collection of electroencephalographic (EEG) data along with other questionnaire measures. Consistent with the university community from which participants were recruited, the racial composition of this sample was $81.8 \%$ Caucasian and 7.6\% African American, with the remaining $10.6 \%$ self-identifying as biracial or other. Procedures for this study were approved by the university's institutional review board (HSC Number 2017.2025) and all participants provided verbal and written consent.

\section{Criterion Measures}

\section{Self-Report}

Externalizing Spectrum Inventory-Brief Form (ESI-BF; Patrick, Kramer, et al., 20/3). The ESI-BF is a 160-item questionnaire developed to index problem behaviors and traits associated with externalizing psychopathology. The measure includes item-based scales for indexing three higher order factors, termed General Disinhibition $(\alpha=$ $.88)$, Callous Aggression $(\alpha=.92)$, and Substance Abuse $(\alpha=.93)$.

Crime and Analogous Behavior Scale (CAB; Lynam, Whiteside, \& Jones, 1999). The CAB is a self-report measure of violent and nonviolent antisocial behavior (nine items; $\alpha=$ .74), and substance abuse (seven items; $\alpha=.74$ ). Participants respond yes/no regarding their engagement in each listed behavior.

Multidimensional Personality Questionnaire-Brief Form (MPQ-BF; Patrick, Curtin, \& Tellegen, 2002). The MPQ-BF is a 155 -item self-report inventory of general personality that yields scores on both lower order trait scales (WellBeing, Social Potency, Achievement, Social Closeness, Stress Reaction, Alienation, Aggression, Control, Harm Avoidance, Traditionalism, and Absorption; Cronbach's $\alpha$ ranging from .68 to .98) and higher order personality dimensions (Positive Emotionality, $\alpha=$.78; Negative Emotionality, $\alpha=.98$; Constraint, $\alpha=.81$ ).

Inventory of Depression and Anxiety Symptoms (IDAS; Watson et al., 2007). The IDAS is a 64-item self-report measure of mood and anxiety disorder symptoms. The measure includes 10 specific symptom scales (Suicidality, Lassitude, Insomnia, Appetite Loss, Appetite Gain, Ill Temper, Well-Being, Panic, Social Anxiety, Traumatic Intrusions; Cronbach's $\alpha$ ranging from .76 to .88) along with two broad pathology scales: General Depression $(\alpha=.89)$ and Dysphoria $(\alpha=.91)$.

Beck Scale for Suicide Ideation (BSS; Beck \& Steer, I99I). The BSS is a well-validated 21-item inventory $(\alpha=.79)$ designed to assess suicidality along a spectrum, including items directed at assessing ideation, plans, access to means, and past attempts.

Trait Fear Inventory (Tf-44; Kramer, Patrick, Hettema, Moore, \& Sawyers, 2017). Variations in fearfulness were assessed using a 44-item inventory (Cronbach's $\alpha=.96$ ). This measure was developed to index dispositional fear based on structural modeling research demonstrating a general "fear/fearlessness" factor underlying established scale measures of this trait construct (Kramer, Patrick, Krueger, \& Gasperi, 2012).

\section{Physiological Response}

Experimental task stimuli. The visual oddball task consisted of a modified version of the "rotated heads" paradigm designed by Begleiter et al. (1984). Participants viewed stimuli of three types: simple nontargets (simple ovals); targets (line-drawn "heads"), calling for a buttonpress response; and novel nontargets (photo-image faces). The simple nontarget stimuli appeared $70 \%$ of the time (168 out of 240 total trials), and the target and novel stimuli each appeared $15 \%$ of the time (36 trials each).

Stimulus delivery and recording procedure. Participants viewed the task stimuli on a 21 -inch monitor while seated in a comfortable chair with a button-response box positioned on their lap. All stimuli (ovals, heads, and faces) were presented within a dark gray rectangle against a black background. The stimuli were presented for $100 \mathrm{~ms}$ each, separated by intervals of 4 to 5 seconds.

Physiological data recording and reduction parameters. EEG activity was recorded using a 128-channel head cap that positioned sintered $\mathrm{Ag}-\mathrm{AgCl}$ electrodes according to the nonstandard layout (NSL; Compumedics Neuroscan, Inc., El Paso, TX) system. The raw EEG signal was continuously recorded at a rate of $1000 \mathrm{~Hz}$ and band-pass filtered online from 0.05 to $200 \mathrm{~Hz}$ using a Neuroscan Synamps system. This raw data were referenced online to NSL electrode 64, analogous to electrode $\mathrm{CPz}$ in the 10-20 system. The filtered raw EEG data were parsed offline into epochs extending from $1,000 \mathrm{~ms}$ before to 2,000 $\mathrm{ms}$ after stimulus onset, and then averaged across trials within condition (nontarget, target, novel). The average epoched signal was then baseline-corrected by subtracting from each aggregate time point the mean amplitude of EEG activity across a $500 \mathrm{~ms}$ prestimulus interval. Trials with eyeblinks, eye movements, or muscle potentials exceeding $75 \mu \mathrm{V}$ at any 
Table 4. Correlations for Self-Report Externalizing Traits and Behaviors with Scores on the IMAGEN-DIS Scale in an Undergraduate Sample.

\begin{tabular}{lc}
\hline Criterion measure & Disinhibition \\
\hline ESI-BF & $.79 * *$ \\
General Disinhibition & $.63^{* *}$ \\
Callous Aggression & $.47^{* *}$ \\
Substance Use & \\
CAB & $.59^{* *}$ \\
Antisocial Behavior & $.48^{* *}$ \\
Substance Abuse
\end{tabular}

Note. $n=66$. IMAGEN-DIS = IMAGEN-Disinhibition; ESI-BF = Externalizing Spectrum Inventory-Brief Form; $C A B=$ Crime and Analogous Behavior Scale. $* * p<.001$.

Table 5. Correlations for Self-Report Measures of Personality and Internalizing Psychopathology with Scores on the IMAGENDIS Scale in an Undergraduate Sample.

\begin{tabular}{lc}
\hline Criterion measure & Disinhibition \\
\hline MPQ-BF $(n=65)$ & \\
Positive Emotionality & -.02 \\
Well-Being & -.03 \\
Social Potency & .17 \\
Achievement & $-.27^{*}$ \\
Social Closeness & -.04 \\
Negative Emotionality & $.47^{* *}$ \\
Stress Reaction & $.27^{*}$ \\
Alienation & $.37^{*}$ \\
Aggression & $.62^{* *}$ \\
Constraint & $-.55^{* *}$ \\
Control & $-.59^{* *}$ \\
Harm Avoidance & $-.34^{*}$ \\
Traditionalism & -.24 \\
Absorption & .08 \\
IDAS & \\
General Depression & .20 \\
Dysphoria & .24 \\
BSS & $.26 *$ \\
TF-44 & -.14 \\
\hline
\end{tabular}

Note. $n=66$. MPQ-BF = Multidimensional Personality QuestionnaireBrief Form; IDAS = Inventory of Depression and Anxiety Symptoms; BSS $=$ Beck Scale for Suicide Ideation; TF-44 = Trait Fear Inventory. $*_{p}<.05 . * * p<.001$.

electrode were excluded from averaging. P3 amplitude was quantified as the maximum peak voltage within a window of $250 \mathrm{~ms}$ to $500 \mathrm{~ms}$ following stimulus onset. In line with prior research on $\mathrm{P} 3$ as a neural indicator, which has generally focused on the midline parietal $(\mathrm{Pz})$ site, $\mathrm{P} 3$ response assessed at NSL electrode site 66 (analogous to Pz) was used in all analyses. A waveform plot for the $\mathrm{P} 3$ response to target stimuli in the sample as a whole (accompanied by a headmap depicting its scalp topography) is included in the online Supplement to this article.

\section{Results}

Within the undergraduate participant sample, the IMAGENDIS scale displayed acceptable internal consistency $(\alpha=.74)$. Results from analyses examining associations between IMAGEN-DIS scores and externalizing traits and behaviors in the undergraduate sample are shown in Table 4. Consistent with hypotheses, IMAGEN-DIS was highly correlated with the General Disinhibition factor scale of the ESI-BF ( $r=$ .79). At the bivariate level, the Callous Aggression and Substance Use factor scales also showed appreciable correlations with the IMAGEN-DIS scale; however, when all three ESI-BF factor scales were entered into a regression model predicting IMAGEN-DIS, the strongest predictor was the General Disinhibition scale $(\beta=.58, p<.001)$, with Callous Aggression maintaining significant but weakened prediction $(\beta=.26, p<.01)$, and the Substance Abuse scale no longer significant $(\beta=.15, p>.01)$. Also consistent with study hypotheses, IMAGEN-DIS scale scores also correlated significantly with the Antisocial Behavior and Substance Abuse subscales of the CAB.

Associations between scores on the IMAGEN-DIS scale and self-report measures of personality and internalizing psychopathology are also shown in Table 5. IMAGEN-DIS was most associated with traits in the Negative Emotionality and Constraint domains of the MPQ-BF. In particular, individuals high in IMAGEN-DIS showed high levels of Aggression and Alienation, along with low levels of Control and Harm Avoidance. IMAGEN-DIS was not significantly related to internalizing psychopathology as defined by the broad IDAS scales (General Depression and Dysphoria); however, it was significantly associated with the Ill Temper $(r=.53, p<.001)$, Panic $(r=.32, p<.01)$, and Appetite Gain $(r=.30, p<.05)$ symptom scales. As a further test of discriminant validity, and in line with hypotheses, the IMAGEN-DIS scale was not related to trait fear in the undergraduate sample. However, consistent with prior work (Buchman-Schmitt et al., 2017; Venables et al., 2015), it did show a modest but significant positive $r$ with suicidal tendencies as indexed by the BSS.

Consistent with our a priori hypothesis, the IMAGENDIS scale showed a significant negative association with amplitude of P3 response to target "head" stimuli in the oddball task ( $r=-.34, p<.01$; see Figure 1$)$-at a level similar to that for the ESI Disinhibition scale $(r=-.31)$. Higher scores on the IMAGEN-DIS factor scale were also associated with reduced $\mathrm{P} 3$ response to novel picture stimuli within this task $(r=-.30, p<.05){ }^{2} \mathrm{~A}$ follow-up regression analysis was used to test whether the newly developed IMAGEN-DIS scale accounted for the same variance in $\mathrm{P} 3$ response to target stimuli as the well-validated ESI-Disinhibition scale. The 


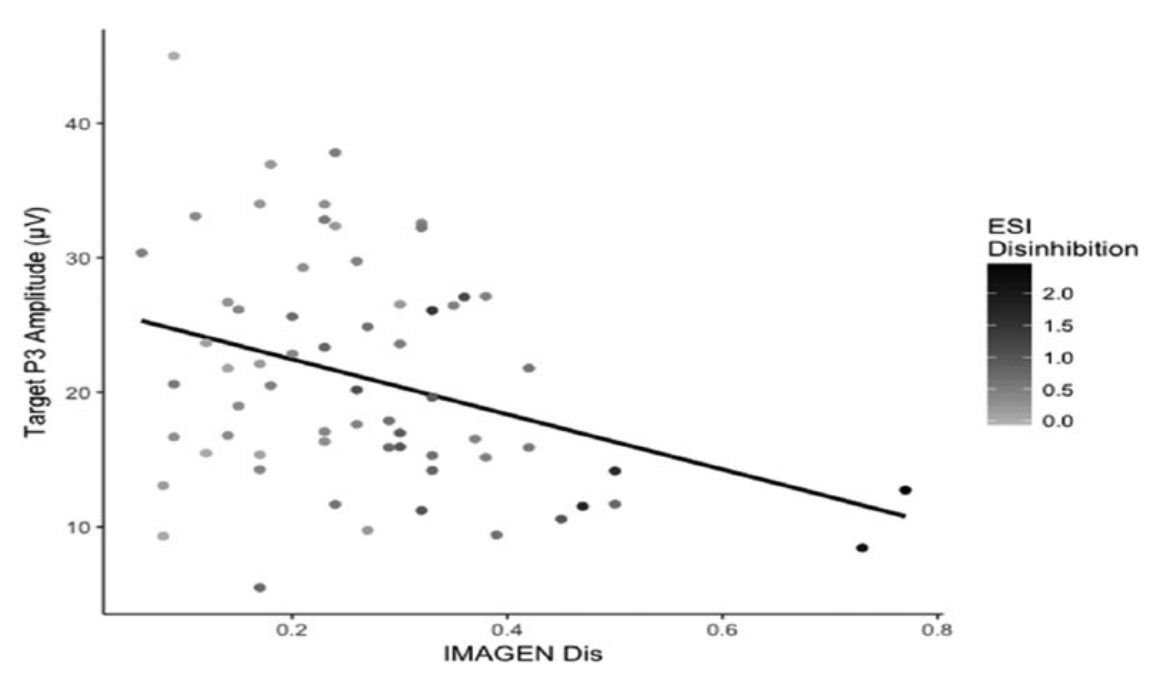

Figure I. Scatterplot of the association between IMAGEN-DIS scale score and peak P3 response to oddball-task target stimuli at electrode $66(\mathrm{Pz} ; r=-.34, p<.0 \mathrm{I})$, with data points shaded to reflect corresponding magnitude of ESI Disinhibition scale score for each participant.

Note. ESI = Externalizing Spectrum Inventory.

model included both IMAGEN-DIS and ESI-Disinhibition as predictors of target $\mathrm{P} 3$ response amplitude at scalp site $\mathrm{Pz}$; the overall regression model was significant $(R=.37$, $p<.05)$, but neither scale evidenced significant independent prediction $(\beta \mathrm{s}=-.14$ and -.25 , respectively, $p \mathrm{~s}>.05)$-indicating that their mutual association with P3 response was attributable to their shared variance.

\section{Discussion}

One major aim in the current work was to develop and validate an effective scale measure of trait disinhibition using questionnaire items available in the IMAGEN study. A second and related aim was to test the hypothesis, based on extensive prior work delineating the nomological network of the construct of trait disinhibition (externalizing proneness), that this scale measure would interface with the domain of neurophysiology - that is, correlate with a wellestablished brain indicator of disinhibitory tendencies (i.e., P3 ERP response) in an independent participant sample. Our broader aim was to demonstrate the potential utility of this scale measure as a referent for advancing a neuroclinical approach to the assessment of disinhibitory liability (Kwako et al., 2016) through further work with the IMAGEN data set.

The scale development phase of this study produced a final IMAGEN-DIS scale with items derived from contentrelevant subscales of different inventories available in the IMAGEN data set (see Table 1). By using a construct-rating approach to identify items to compose the scale, the resultant IMAGEN-DIS scale was expected to share the nomological network of the trait disinhibition construct and relate in expected ways with variables from the domains of clinical problems and neurophysiological response that are part of this network. Our findings for criterion measures in both the IMAGEN adolescent sample and the undergraduate adult sample were consistent with this perspective.

Within the adolescent sample, the IMAGEN-DIS scale showed acceptable internal consistency at Time 1 and Time 2 as well as temporal stability across the two assessment times. Evidence for the construct validity of the IMAGENDIS scale operationalization of trait disinhibition was demonstrated through the finding of hypothesized relations with DAWBA-based clinical ratings of DSM- $I V$ defined disruptive disorders (CD, ODD, ADHD), and substance use/abuse symptoms as indexed by the AUDIT and ESPAD, at both Time 1 and Time 2. In addition, consistent with evidence that general proneness toward impulse-control problems is indicative of disinhibitory liability, the IMAGEN-DIS scale demonstrated strong significant associations $(r \mathrm{~s} \sim 4)$ with the EXT composite variable at both Time 1 and Time 2 . Furthermore, and of particular importance, IMAGEN-DIS scores at Time 1 predicted relations with these criterion measures of externalizing problems at Time 2 - over and above levels of these problems exhibited at Time 1. Taken together, these findings lay a foundation for future studies using scores on the IMAGEN-DIS scale at Time 1 as a reportbased psychological index of disinhibitory liability that can serve as a referent for identifying neurophysiological and task-behavioral indicators of this dispositional liability. 
As expected given the use of a construct-based approach to scale development that has proven effective in prior work with adults (e.g., Brislin et al., 2015; Hall et al., 2014; Sellbom et al., 2016), the IMAGEN-DIS scale showed acceptable internal consistency in the undergraduate sample, indicating that the items of the scale cohere together similarly at these two distinct ages $(M \mathrm{~s}=14.4$ and 19.5 years, respectively). These results are particularly exciting in the context of the IMAGEN study, as they suggest that the IMAGEN-DIS scale can serve as an effective proxy measure of trait disinhibition across successive waves of data collection. In addition, the associations between the IMAGEN-DIS scale and the factor scales of the ESI-BF in this older participant sample provide further support for its construct validity. While IMAGEN-DIS scores showed significant bivariate $r$ s with scores on all three of the ESI-BF factors, it correlated most highly with ESI-Disinhibition, and this pattern was accentuated when regression analyses were used to account for covariance among the ESI-BF factors. Similarly, and as hypothesized, the IMAGEN-DIS scale was positively associated with self-reported antisocial behavior and substance abuse (as indexed by the $\mathrm{CAB}$ ) in the undergraduate participant sample.

The IMAGEN-DIS scale also showed significant correlations with trait scales of the MPQ-BF that relate to higher order personality factors of Negative Emotionality and Constraint (cf. Krueger, 1999). Notably, the pattern of $r$ s for the IMAGEN-DIS scale with specific MPQ-BF traits paralleled those reported for the ESI Disinhibition scale in prior large- $N$ undergraduate work (Drislane, Patrick, \& Arsal, 2014, Table 1): It correlated strongly with MPQ-BF Aggression (+) and Control $(-)$, and more modestly with MPQ-BF Alienation (+), Harm Avoidance (-), Stress Reaction (+), and Achievement $(-)$. The IMAGEN-DIS scale was weakly and nonsignificantly related to general internalizing psychopathology as indexed by the IDAS, but it did demonstrate a significant positive correlation with the IDAS Panic scale, consistent with the association between ESI Disinhibition and symptoms of panic disorder reported by Nelson, Strickland, Krueger, Arbisi, and Patrick (2016), and a somewhat weaker positive $r$ with the Appetite Gain scale of the IDAS. Consistent with prediction based on prior work (Nelson et al., 2016), the IMAGEN-DIS scale was uncorrelated with general fearfulness as indexed by the TF-44.

The IMAGEN-DIS scale also demonstrated a significant association as predicted with neurophysiological response. Specifically, individuals who scored higher on the IMAGEN-DIS scale demonstrated reduced amplitude of P3 response to target stimuli in the oddball task (Patrick, Venables, et al., 2013; Yancey et al., 2013), and also to novel stimuli within this task (Nelson et al., 2011; Patrick, Venables, et al., 2013). These associations and the magnitude of the effects seen are consistent with prior research that has examined relations between P3 amplitude and alternative operationalizations of disinhibitory liability (Patrick et al., 2006; Yancey et al., 2013). Indeed, when scores on the ESI-Disinhibition scale, a well-validated selfreport measure of trait disinhibition, were entered into a regression model alongside IMAGEN-DIS scores as predictors of $\mathrm{P} 3$ amplitude to oddball-target stimuli, the overall model $R$ was significant but neither scale variable showed a unique contribution to prediction. These results indicate that the IMAGEN-DIS scale captures the systematic variance in ESI-Disinhibition scores that relates to P3 response amplitude.

Importantly, prior twin-study research has demonstrated that the variance in ESI-Disinhibition scores that relates to $\mathrm{P} 3$ response is entirely attributable to genetic influence, and that this shared component of genetic variance overlaps in turn with genetic variance in externalizing disorder symptoms (Yancey et al., 2013). Given other evidence for a genetic basis to the relationship between disinhibitory tendencies and P3 amplitude (Hicks et al., 2007), it plausible to suppose that the correlations observed for the IMAGENDIS scale with P3 response and externalizing symptoms in the second study sample reflect heritable variance in common among these three measured variables (i.e., latent disinhibitory liability). However, this possibility needs to be directly evaluated in a larger sized twin data set.

\section{Limitations and Future Directions}

Some limitations of the current work warrant mention. First, the pool of items for developing the new trait disinhibition scale was constrained by the preexisting nature of the IMAGEN database-i.e., only items administered within the study could be used to adequately represent the target construct. Mitigating this concern, results from the two study samples indicate that the available item pool provided effective coverage of the construct and came together to form a psychometrically valid operationalization of trait disinhibition.

The modest size of the undergraduate participant sample that was used to further validate the IMAGEN-DIS scale ( $n$ $=66$ ) is a further limitation. Although findings from this second validation sample were largely consistent with hypotheses based on extensive prior research, further validation work using a larger sample and a wider array of criterion measures would be helpful for further establishing the construct validity of the scale. In the future, the IMAGEN-DIS scale could be further evaluated by examining its loading on a latent disinhibition factor alongside other established scale measures of this construct (see Drislane \& Patrick, 2017). Future work with twin-samples would be also be valuable for clarifying the degree of etiologic overlap between IMAGEN-DIS scores and other indicators of disinhibition including alternative scale measures, interview-assessed externalizing problems, neurophysiological variables 
including P3, and cognitive-performance measures (see Young et al., 2009).

The current work provides evidence for the validity of the IMAGEN-DIS scale as a report-based proxy measure for indexing underlying dispositional liability for impulse-control problems in this unique large-scale data set. Along with self-report, clinical-diagnostic, and task-performance measures, neuroimaging data of various types (i.e., structural MRI, resting-state and task-related functional MRI, diffusion tensor imaging) are available for participants in the IMAGEN project. The current work sets the stage for further systematic work with data from this project directed at identifying neuroimaging and task-performance variables that correlate with scale-assessed disinhibition, and that can be further investigated as indicators of heritable risk for externalizing psychopathology (i.e., as endophenotypes; Gottesman \& Gould, 2003). The large sample size of the IMAGEN study lends itself to a split-half cross-validation design in order to maximize the replicability of identified indicators. In addition, the longitudinal nature of the study design makes it possible to examine predictive relations for brain and behavioral indicators of trait disinhibition with externalizing problems at later time points, controlling for current problems (as was done for the IMAGEN-DIS scale in the adolescent sample of the current work).

The current findings and their research implications connect to recent writings in the addictions field encouraging investigators to move toward a process-driven, as opposed to outcome-oriented, approach to understanding problematic alcohol and other substance use. For example, Kwako et al. (2016) called for research on addiction-related processes to be coordinated (harmonized) through collection of a standard battery of measures from different assessment modalities including self-report, behavioral performance, and neurophysiology - termed the Addiction Neuroclinical Assessment battery. One of the three core process domains they identified as a target for the Addiction Neuroclinical Assessment was executive function, corresponding to inhibitory control capacity (inhibition-disinhibition) as conceptualized here.

The current work suggests a strategy for progressing toward an integrative assessment of this core addictionrelated capacity using data from the IMAGEN project and other existing large-scale, multidomain data sets. The strategy involves creating effective scale measures of this construct from available questionnaire items that can be used both as referents for identifying neural and behavioral indicators of inhibitory control (executive function) capacity within a given data set, and for harmonizing that data set with other complementary data sets. In this way, the field can advance toward multidomain assessments of this core dispositional attribute that demonstrate reliable associations with variables of interest in domains of neurophysiology and behavioral performance as well as clinical adjustment.

\section{Authors' Note}

The views, opinions, and/or findings contained in this report are those of the authors and shall not be construed as an official Department of the Army position, policy, or decision, unless so designated by other documents.

\section{Declaration of Conflicting Interests}

The author(s) declared the following potential conflicts of interest with respect to the research, authorship, and/or publication of this article: Dr. Banaschewski has served as an advisor or consultant to Bristol-Myers Squibb, Desitin Arzneimittel, Eli Lilly, Medice, Novartis, Pfizer, Shire, UCB, and Vifor Pharma; he has received conference attendance support, conference support, or speaking fees from Eli Lilly, Janssen McNeil, Medice, Novartis, Shire, and UCB; and he is involved in clinical trials conducted by Eli Lilly, Novartis, and Shire; the present work is unrelated to these relationships.

\section{Funding}

The author(s) disclosed receipt of the following financial support for the research, authorship, and/or publication of this article: The project on which this article is based received support from the following sources: the European Union-funded FP6 Integrated Project IMAGEN (Reinforcement-related behavior in normal brain function and psychopathology; LSHM-CT-2007-037286), the Horizon 2020 funded ERC Advanced Grant "STRATIFY" (Brain network-based stratification of reinforcement-related disorders; 695313), ERANID (Understanding the Interplay between Cultural, Biological and Subjective Factors in Drug Use Pathways; PR-ST-0416-10004), BRIDGET (JPND: BRain Imaging, cognition Dementia and next generation GEnomics; MR/N027558/1), the FP7 projects IMAGEMEND (602450; IMAging GEnetics for MENtal Disorders) and MATRICS (603016), the Innovative Medicine Initiative Project EU-AIMS (115300-2), the Medical Research Council Grant "c-VEDA" (Consortium on Vulnerability to Externalizing Disorders and Addictions; MR/N000390/1), the Swedish Research Council FORMAS, the Medical Research Council, the National Institute for Health Research (NIHR) Biomedical Research Centre at South London and Maudsley NHS Foundation Trust and King's College London, the Bundesministeriumfür Bildung und Forschung (BMBF Grants 01GS08152; 01EV0711; eMED SysAlc01ZX1311A; Forschungsnetz AERIAL), the Deutsche Forschungsgemeinschaft (DFG Grants SM 80/7-1, SM 80/7-2, SFB 940/1). Further support for work reported in this article was provided by the following sources: ANR (project AF12-NEUR0008-01, WM2NA, and ANR-12-SAMA-0004), the Fondation de France, the Fondation pour la Recherche Médicale, the Mission Interministérielle de Luttecontre-les-Drogues-et-les-Conduites-Addictives (MILDECA), the Assistance-Publique-Hôpitaux-de-Paris and INSERM (interface grant), Paris Sud University IDEX 2012; the National Institutes of Health, United States (Axon, Testosterone, and Mental Health during Adolescence; RO1 MH085772-01A1), and NIH Consortium grant U54 EB020403, supported by a cross-NIH alliance that funds Big Data to Knowledge Centres of Excellence; the National Science Foundation Graduate Research Fellowship program, under Grant No. 952090; and Grant W911NF-14-1-0018 from the U.S. Army. 


\section{Notes}

1. Research in the field of schizophrenia suggests that blunted P3 amplitude may also be an endophenotype for psychosis (e.g., Bramon et al., 2005). Of note, associations for P3 with schizophrenia are most evident when stimuli are presented in the auditory modality (Earls, Curran, \& Mittal, 2016), as opposed to visually in the case of externalizing disorders. However, further research is needed to determine whether reduced $\mathrm{P} 3$ response in relation to conditions of these two types (psychotic, externalizing) reflects common or distinct cognitive deficits. These findings serve as a reminder that brain response indicators contain differing sources of variance that may underlie associations with different psychological phenotypes (for a detailed discussion of this issue, see Perkins et al., 2017).

2. When P3 responses for both target and novel stimuli were entered together into a regression model predicting IMAGENDIS scores, the overall model was significant $(R=.34, p$ $<.05$ ), with target P3 contributing uniquely at a trend-level (B $=-.37, p=.09)$ and novel $\mathrm{P} 3$ showing no unique predictive contribution $(\mathrm{B}=.04, p=.86)$. The implication, consistent with our past work (Nelson et al., 2011; Patrick, Venables, et al., 2013), is that different variants of $P 3$ response overlap in their associations with trait disinhibition.

\section{Supplemental Material}

The online supplementary material is available at http://journals. sagepub.com/doi/suppl/10.1177/1073191118759748.

\section{ORCID iDs}

Dimitri Papadopoulos Orfanos (iD https://orcid.org/0000-00021242-8990

Juliane H. Fröhner (iD https://orcid.org/0000-0002-8493-6396

Jens Foell iD https://orcid.org/0000-0002-2089-094X

\section{References}

Abram, S. V., Wisner, K. M., Grazioplene, R. G., Krueger, R. F., MacDonald, A. W., III, \& DeYoung, C. G. (2015). Functional coherence of insula networks is associated with externalizing behavior. Journal of Abnormal Psychology, 124, 1079-1091.

Allen, J. P., Litten, R. Z., Fertig, J. B., \& Babor, T. (1997). A review of research on the Alcohol Use Disorders Identification Test (AUDIT). Alcoholism: Clinical \& Experimental Research, 21, 613-619.

Allport, G. W. (1937). Personality: a psychological interpretation. Oxford, England: Holt.

American Psychiatric Association. (2000). Diagnostic and statistical manual of mental disorders (4th ed., text rev.). Washington, DC: Author.

Barkley, R. A. (1997). Behavioral inhibition, sustained attention, and executive functions: constructing a unifying theory of ADHD. Psychological bulletin, 121(1), 65-94.

Beck, A. T., \& Steer, R. A. (1991). BSS: Beck Scale for Suicide Ideation. San Antonio, TX: Psychological Corporation.

Begleiter, H., Porjesz, B., Bihari, B., \& Kissin, B. (1984). Eventrelated brain potentials in boys at risk for alcoholism. Science, 225, 1493-1496.
Bramon, E., McDonald, C., Croft, R. J., Landau, S., Filbey, F., Gruzelier, J. H., . . Murray, R. M. (2005). Is the P300 wave an endophenotype for schizophrenia? A meta-analysis and a family study. NeuroImage, 27, 960-968.

Brislin, S. J., Drislane, L. E., Smith, S. T., Edens, J. F., \& Patrick, C. J. (2015). Development and validation of triarchic psychopathy scales from the Multidimensional Personality Questionnaire. Psychological Assessment, 27, 838-851.

Buchman-Schmitt, J. M., Brislin, S. J., Venables, N. C., Joiner, T. E., \& Patrick, C. J. (2017). Trait liabilities and specific promotive processes in psychopathology: The example of suicidal behavior. Journal of affective disorders, 216, 100-108.

Cloninger, C. R. (1999). The Temperament and Character Inventory-Revised. St Louis, MO: Center for Psychobiology of Personality, Washington University.

Conrod, P. J., \& Woicik, P. (2002). Validation of a four-factor model of personality risk for substance abuse and examination of a brief instrument for assessing personality risk. Addiction Biology, 7, 329-346.

Costa, P. T., Jr., \& McCrae, R. R. (1992). Revised NEO Personality Inventory (NEO PI-R) and NEO Five-Factor Inventory (NEO-FFI) professional manual. Odessa, FL: Psychological Assessment Resources.

Drislane, L. E., \& Patrick, C. J. (2017). Integrating alternative conceptions of psychopathic personality: A latent variable model of triarchic psychopathy constructs. Journal of Personality Disorders, 31, 110-132.

Drislane, L. E., Patrick, C. J., \& Arsal, G. (2014). Clarifying the content coverage of differing psychopathy inventories through reference to the Triarchic Psychopathy Measure. Psychological Assessment, 26, 350-362.

Earls, H. A., Curran, T., \& Mittal, V. (2016). A meta-analytic review of auditory event-related potential components as endophenotypes for schizophrenia: Perspectives from firstdegree relatives. Schizophrenia Bulletin, 42, 1504-1516.

Eysenck, H. J. (1960). Classification and the problem of diagnosis. In Eysenck, H. J. (Ed.), Handbook of abnormal psychology(pp. 1-31). London, UK: Pitman.

Foell, J., Brislin, S. J., Strickland, C. M., Seo, D., Sabatinelli, D., \& Patrick, C. J. (2016). Externalizing proneness and brain response during pre-cuing and viewing of emotional pictures. Social Cognitive and Affective Neuroscience, 11, $1102-1110$

Goodman, R. (2001). Psychometric properties of the Strengths and Difficulties Questionnaire. Journal of the American Academy of Child \& Adolescent Psychiatry, 40, 1337-1345.

Goodman, R., Ford, T., Richards, H., Gatward, R., \& Meltzer, H. (2000). The Development and Well-Being Assessment: Description and initial validation of an integrated assessment of child and adolescent psychopathology. Journal of Child Psychology and Psychiatry, 41, 645-655.

Gottesman, I. I., \& Gould, T. D. (2003). The endophenotype concept in psychiatry: etymology and strategic intentions. American Journal of Psychiatry, 160(4), 636-645.

Hall, J. R., Bernat, E. M., \& Patrick, C. J. (2007). Externalizing psychopathology and the error-related negativity. Psychological science, 18(4), 326-333.

Hall, J. R., Drislane, L. E., Patrick, C. J., Morano, M., Lilienfeld, S. O., \& Poythress, N. G. (2014). Development and validation of 
Triarchic construct scales from the Psychopathic Personality Inventory. Psychological Assessment, 26, 447-461.

Hicks, B. M., Bernat, E. M., Malone, S. M., Iacono, W. G., Patrick, C. J., Krueger, R. F., \& McGue, M. (2007). Genes mediate the association between P300 amplitude and externalizing psychopathology. Psychophysiology, 44, 98-105.

Iacono, W. G., Carlson, S. R., Malone, S. M., \& McGue, M. (2002). P3 event-related potential amplitude and the risk for disinhibitory disorders in adolescent boys. Archives of general psychiatry, 59(8), 750-757.

Kim-Cohen, J., Caspi, A., Moffitt, T. E., Harrington, H., Milne, B. J., \& Poulton, R. (2003). Prior juvenile diagnoses in adults with mental disorder: Developmental follow-back of a prospective-longitudinal cohort. Archives of General Psychiatry, 60, 709-717.

Kozak, M. J., \& Cuthbert, B. N. (2016). The NIMH Research Domain Criteria initiative: Background, issues, and pragmatics. Psychophysiology, 53, 286-297.

Kramer, M. D., Patrick, C. J., Hettema, J. M., Moore, A. A., \& Sawyers, C. (2017). Dispositional fear and fearlessness: Development and initial validation of a model-based scale measure. Manuscript submitted for publication.

Kramer, M. D., Patrick, C. J., Krueger, R. F., \& Gasperi, M. (2012). Delineating physiologic defensive reactivity in the domain of self-report: Phenotypic and etiologic structure of dispositional fear. Psychological Medicine, 42, 1305-1320.

Krueger, R. F. (1999). Personality traits in late adolescence predict mental disorders in early adulthood: A prospective-epidemiological study. Journal of Personality, 67, 39-65.

Krueger, R. F., Hicks, B. M., Patrick, C. J., Carlson, S. R., Iacono, W. G., \& McGue, M. (2002). Etiologic connections among substance dependence, antisocial behavior, and personality: modeling the externalizing spectrum. Journal of Abnormal Psychology, 111, 411-424.

Krueger, R. F., Markon, K. E., Patrick, C. J., Benning, S. D., \& Kramer, M. D. (2007). Linking antisocial behavior, substance use, and personality: An integrative quantitative model of the adult externalizing spectrum. Journal of Abnormal Psychology, 116, 645-666.

Kwako, L. E., Momenan, R., Litten, R. Z., Koob, G. F., \& Goldman, D. (2016). Addictions neuroclinical assessment: A neuroscience-based framework for addictive disorders. Biological Psychiatry, 80, 179-189.

Lynam, D. R., Whiteside, S., \& Jones, S. (1999). Self-reported psychopathy: A validation study. Journal of Personality Assessment, 73, 110-132.

Morgan, A. B., \& Lilienfeld, S. O. (2000). A meta-analytic review of the relation between antisocial behavior and neuropsychological measures of executive function. Clinical psychology review, 20(1), 113-136.

National Research Council (2015). Measuring human capabilities: An agenda for basic research on the assessment of individual and group performance potential for military accession. Committee on Measuring Human Capabilities, Division of Behavioral and Social Sciences and Education. Washington, DC: National Academy of Sciences.

Nelson, L. D., Patrick, C. J., \& Bernat, E. M. (2011). Operationalizing proneness to externalizing psychopathology as a multivariate psychophysiological phenotype. Psychophysiology, 48(1), 64-72.
Nelson, L. D., Strickland, C., Krueger, R. F., Arbisi, P. A., \& Patrick, C. J. (2016). Neurobehavioral traits as transdiagnostic predictors of clinical problems. Assessment, 23, 75-85.

Newcomb, M. D., Huba, G. J., \& Bentler, P. M. (1981). A multidimensional assessment of stressful life events among adolescents: Derivation and correlates. Journal of Health and Social Behavior, 22, 400-415.

Patrick, C. J., Bernat, E., Malone, S. M., Iacono, W. G., Krueger, R. F., \& McGue, M. K. (2006). P300 amplitude as an indicator of externalizing in adolescent males. Psychophysiology, 43, 84-92.

Patrick, C. J., Curtin, J. J., \& Tellegen, A. (2002). Development and validation of a brief form of the Multidimensional Personality Questionnaire. Psychological Assessment, 14, 150-163.

Patrick, C. J., \& Drislane, L. E. (2015). Triarchic model of psychopathy: Origins, operationalizations, and observed linkages with personality and general psychopathology. Journal of personality, 83(6), 627-643.

Patrick, C. J., Fowles, D. C., \& Krueger, R. F. (2009). Triarchic conceptualization of psychopathy: Developmental origins of disinhibition, boldness, and meanness. Development and Psychopathology, 21, 913-938.

Patrick, C. J., \& Hajcak, G. (2016). RDoC: Translating promise into progress. Psychophysiology, 53(3), 415-424.

Patrick, C. J., Kramer, M. D., Krueger, R. F., \& Markon, K. E. (2013). Optimizing efficiency of psychopathology assessment through quantitative modeling: Development of a brief form of the Externalizing Spectrum Inventory. Psychological Assessment, 25, 1332-1348.

Patrick, C. J., Venables, N. C., Yancey, J. R., Hicks, B. M., Nelson, L. D., \& Kramer, M. D. (2013). A construct-network approach to bridging diagnostic and physiological domains: Application to assessment of externalizing psychopathology. Journal of Abnormal Psychology, 122, 902-916.

Perkins, E. R., Yancey, J. R., Drislane, L. E., Venables, N. C., Balsis, S., \& Patrick, C. J. (2017). Methodological issues in the use of individual brain measures to index trait liabilities: The example of noise-probe P3. International Journal of Psychophysiology, 111, 145-155.

Pihl, R. O., Peterson, J., \& Finn, P. R. (1990). Inherited predisposition to alcoholism: Characteristics of sons of male alcoholics. Journal of Abnormal Psychology, 99(3), 291-301.

Sellbom, M., Drislane, L. E., Johnson, A. K., Goodwin, B. E., Phillips, T. R., \& Patrick, C. J. (2016). Development and validation of MMPI-2-RF Scales for indexing triarchic psychopathy constructs. Assessment, 23(5), 527-543.

Schumann, G., Loth, E., Banaschewski, T., Barbot, A., Barker, G., Büchel, C., . . Struve, M. (2010). The IMAGEN study: Reinforcement-related behaviour in normal brain function and psychopathology. Molecular Psychiatry, 15, 11281139.

Tellegen, A. (1982). Brief manual for the Multidimensional Personality Questionnaire. Unpublished manuscript, University of Minnesota, Minneapolis.

Tellegen, A. (1985). Structures of mood and personality and their relevance to assessing anxiety, with an emphasis on selfreport. In A. H. Tuma \& J. D. Maser (Eds.), Anxiety and the anxiety disorders (pp. 681-706). Hillsdale, NJ, US: Lawrence Erlbaum Associates, Inc. 
Vaidyanathan, U., Patrick, C. J., \& Iacono, W. G. (2011). Patterns of comorbidity among mental disorders: A person-centered approach. Comprehensive Psychiatry, 52, 527-535.

Venables, N. C., Foell, J., Yancey, J. R., Kane, M. J., Engle, R. W., \& Patrick, C. J. (in press). Quantifying inhibitory control as externalizing proneness: A cross-domain model. Clinical Psychological Science.

Venables, N. C., Sellbom, M., Sourander, A., Kendler, K. S., Joiner, T. E., Drislane, L. E., Sillanmäki , L., Elonhieimo, H., Parkkola, K., Multimäki, P., \& Patrick, C. J. (2015). Separate and interactive contributions of weak inhibitory control and threat sensitivity to prediction of suicide risk. Psychiatry Research, 226, 461-466.

Watson, D., O’Hara, M. W., Simms, L. J., Kotov, R., Chmielewski, M., McDade-Montez, E. A., ... Stuart, S. (2007). Development and validation of the Inventory of Depression and Anxiety Symptoms (IDAS). Psychological Assessment, 19, 253-288.
Woicik, P. A., Stewart, S. H., Pihl, R. O., \& Conrod, P. J. (2009). The Substance Use Risk Profile Scale: A scale measuring traits linked to reinforcement-specific substance use profiles. Addictive Behaviors, 34, 1042-1055.

Yancey, J. R., Venables, N. C., Hicks, B. M., \& Patrick, C. J. (2013). Evidence for a heritable brain basis to deviancepromoting deficits in self-control. Journal of Criminal Justice, 41, 309-317.

Young, S. E., Friedman, N. P., Miyake, A., Willcutt, E. G., Corley, R. P., Haberstick, B. C., \& Hewitt, J. K. (2009). Behavioral disinhibition: Liability for externalizing spectrum disorders and its genetic and environmental relation to response inhibition across adolescence. Journal of Abnormal Psychology, 118, 117-130.

Young, S. E., Stallings, M. C., Corley, R. P., Krauter, K. S., \& Hewitt, J. K. (2000). Genetic and environmental influences on behavioral disinhibition. American Journal of Medical Genetics Part A, 96(5), 684-695. 